\title{
Propuesta metodológica para actualizar los estudios catastrales de zonas homogéneas físicas urbanas
}

\author{
Jaime Silva Herrera*
}

Resumen. El presente artículo muestra los resultados de la investigación de nominada "Propuesta Metodológica para Actualizar los Estudios Catastrales de Zonas Homogéneas Físicas Urbanas", desarrollada por el autor como aporte al desarrollo de la gestión catastral colombiana.

Dentro de la actualización de la metodología de zonas homogéneas físicas urba nas, se consideró importante incorporar las variables "Áreas de Actividad" y "tra tamientos urbanísticos", tomados de los planes de Ordenamiento Territoriales Municipales (POT), como variables en gran parte decisorias de los precios del suelo urbano y de expansión urbana.

A lo largo del artículo se presenta el análisis individual e integral, no solo de las dos variables anteriormente señaladas, sino de las otras cinco consideradas dentro de este estudio, que en conjunto ofrecen un estudio más técnico que garantizan unos avalúos catastrales urbanos, más cercanos a la realidad y por ende más justos para los propietarios de los predios. Palabras Clave: áreas de actividad, trata mientos urbanísticos, destinación económica, tipo según destinación económica, servicios, vías, topografía, zonas homogéneas físicas, avalúos catastrales.

* Ingeniero Catastral y Geodesta. MSc. en Administración del Desarrollo Regional. Técnico Científico, IGAC. 


\section{Introducción}

Con la entrada en vigencia de la Ley 14 de 1983, de fortalecimiento de los fiscos municipales, se le otorgó la potestad al Instituto Geográfico Agustín Codazzi de actualizar las metodologías catastrales en todo el país.

Fue así que el IGAC, al finalizar 1984, había diseñado los nuevos estudios, que son los que actualmente siguen vigentes en materia catastral para todo el territorio nacional.

En términos generales la nueva estructura quedó determinada de la siguiente manera:

- RECONOCIMIENTO PREDIAL. Esta etapa prevalece de la metodología anterior y tiene como fundamento la descripción del elemento físico de todos $\mathrm{y}$ cada uno de los predios de un municipio (Determinación de linderos tanto de terreno como de construcción, cálculo de áreas, calificación de construcciones, etc.), como del elemento jurídico. (Indagar y constatar bajo documento la existencia de propietarios o poseedores de los predios, según el caso).

- DISEÑO DE LOS ESTUDIOS DE ZONAS HOMOGÉNEAS FÍSICAS. Es la parte novedosa de la metodología respecto a la anterior, consiste básicamente en validar la homogeneidad de ciertas variables urbanas y/o rurales, según el caso.
En lo urbano, por ejemplo se tienen en cuenta actualmente las siguientes variables como componentes principales de los estudios de zonas homogéneas físicas:

Uso del suelo (Comercial, residencial, dotacional, industrial etc.), servicios (agua, luz, alcantarillado), tipificación de la vivienda (Tipo 1 al tipo 6), vías (pavimentadas, sin pavimentar, peatonales), topografía (plana, ondulada, empinada).

En términos generales se puede definir las zonas homogéneas físicas urbanas, como aquel espacio geográfico con características similares en cuanto a las variables descritas anteriormente.

Una vez demarcadas todas y cada una de las zonas homogéneas físicas, se efectúa una selección dentro de cada una de ellas, de predios representativos de las mismas, dando así inicio al diseño de zonas homogéneas geoeconómicas.

- DISEÑO DE LOS ESTUDIOS DE ZONAS HOMOGÉNEAS GEOECONÓMICAS. Una vez establecidos los diferentes puntos de investigación (predios) dentro de cada una de las zonas homogéneas físicas establecidas, se da inicio a la etapa de investigación económica, que consiste en efectuar una serie de encuestas a personas idóneas del Mercado Inmobiliario de la región, acerca de los precios por metro cuadrado para lo urbano y hectárea para lo rural de todos y cada uno de los terrenos comprendidos en los predios seleccionados dentro de

112 Jaime Silva Herrera 
cada zona homogénea física. Terminadas las encuestas y el respectivo análisis por parte del ejecutante de los estudios, se entra a procesar estadísticamente toda la información recolectada, para así llegar en definitiva a obtener espacios geográficos con el mismo valor por metro cuadrado de terreno, que es lo que se denota catastralmente como zonas homogéneas geoeconómicas.

\section{- DISEÑO DE TABLAS DE CONS-} TRUCCIONES Y ANEXOS. Como bien se conoce, la mayoría de predios soportan sobre sus terrenos diferentes tipos de construcciones, que el catastro al igual que hizo con los terrenos, debe analizar e incorporar a sus estudios. De esta manera en grandes ciudades, se calculan modelos regresionales para cada tipo de construcciones, ya sean residenciales (uni y multifamiliares), comerciales (Hoteles, restaurantes, oficinas, locales, etc., industriales (Bodegas), dotacionales (Colegios, universidades, etc.), que permiten tener gran cobertura de precios por metro cuadrado de construcción, con lo cual el avalúo catastral final se torna más técnico y justo posible.

Complementario a lo anterior, existen ciertas construcciones como piscinas, kioskos, tanques, corrales, establos, cocheras, etc. que el catastro determina también sus valores unitarios.

De esta manera, al integrar el reconocimiento predial, los diseños de zonas homogéneas tanto físicas como geoeconómicas, lo mismo que los resultados obtenidos por los modelos regresionales por cada tipo de construcción más lo obtenidos para valores unitarios de anexos, se obtiene el avalúo catastral de todos y cada uno de los predios comprendidos dentro de la jurisdicción municipal.

Efectuados los avalúos catastrales por las entidades catastrales en cabeza del Agustín Codazzi, estos entes remiten los avalúos a las tesorerías municipales respectivas, donde se les aplican las tarifas que los concejos municipales han aprobado para estos propósitos, dando como resultado el cobro del impuesto predial unificado, que el municipio hace efectivo a todos y cada uno de los propietarios y/o poseedores de los predios situados dentro de su jurisdicción.

Este es el procedimiento que todavía sigue vigente para el cálculo de los avalúos catastrales en Colombia, sin embargo han transcurrido alrededor de 18 años, sin que el proceso se haya actualizado, por ello esta investigación toma la etapa correspondiente al diseño de zonas homogéneas físicas, más exactamente la correspondiente a zonas urbanas y presenta la siguiente propuesta, con el ánimo de darle un valor agregado al proceso catastral colombiano.

\section{Antecedentes de la propuesta}

Con la entrada en vigencia de la Ley 388 de 1997, de ordenamiento territorial y por ende con la expedición de más de la mitad 
de los planes de ordenamiento territoriales municipales (POT) se observó que estos contienen información importante que bien puede ser aprovechada por el Catastro para ser involucrada en sus procesos.

Las normas de uso del suelo urbano hacen parte de la información que trae los POT Municipales y que encajan perfectamente dentro de la metodología de zonas homogéneas físicas urbanas.

Los reglamentos municipales que determinan el uso del suelo, las zonas comerciales, residenciales, los de remodelación urbana privada o pública, etc. influyen sobre los valores de los bienes al fijar las posibilidades de utilización de los predios o más bien los derechos que se pueden ejercer sobre los mismos, pues establecen las normas de alturas, constructibilidad, retiros y aspectos arquitectónicos de las construcciones a edificar en un sector determinado.

Buitrago (2001:98) en su artículo "Análisis de factores formadores del precio de la tierra rural en los municipios contiguos a Bogotá", encuentra que "de esta manera, el atributo artificial uso por norma debe analizarse considerando elementos normativos tales como actividades permitidas o restringidas, tamaños mínimos, índice de ocupación, índice de construcción, densidad de viviendas permitida, entre otros aspectos, que condicionan o potencian el desarrollo de las actividades urbanísticas. En este sentido que existe el impacto de las normas sobre la formación del precio del suelo".
El mismo autor, afirma más adelante que "El cambio de norma afecta directamente el precio de la tierra haciendo que se presenten rentas que lo aumentan o reducen. La renta absoluta urbana aparece cuando se incorpora suelo rural a suelo de expansión urbana, en tanto que las diferenciales surgen al autorizar un mayor aprovechamiento del suelo o ejecutar proyectos que valorizan el terreno; en general, ellas propician la aparición de plusvalías. De igual manera, un predio puede bajar de precio al cambiar la norma de uso, por ejemplo, de agropecuaria a suelos de protección forestal o cuando se amplía el área de la ronda de un río".

Para Rodríguez (2001:110) en su artículo "El valor del suelo y competencia por la ocupación espacial Estudio de Caso: área de influencia Autopista Norte (Calle 200Briceño), encuentra que "con un valor explicativo del $15 \%$ se encuentran las variables correspondientes a uso del suelo por norma. La reglamentación específica de áreas forestales, industriales y comerciales determina en gran proporción el valor del suelo estableciendo el margen de rentabilidad del mismo como factor de producción o como medio de producción natural".

Antes de la entrada en vigencia de los POT Municipales, relativamente eran pocas las zonas urbanas que poseían la norma del uso del suelo aprobadas por los respectivos concejos municipales. Hoy en día con la aprobación de gran parte de los POT Municipales, las zonas urbanas por pequeñas que sean, poseen el estatuto 
de norma del suelo, desapareciendo el obstáculo para ser incorporada por los entes catastrales dentro de sus metodologías, sobre todo los municipios cuya jurisdicción catastral pertenece al Instituto Geográfico Agustín Codazzi, pues el Catastro independiente de la ciudad de Bogotá, así no maneje hasta ahora los denominados tratamientos urbanísticos, si por lo menos ha tomado las áreas de actividad para los procesos de formación como de actualización catastral.

De esta manera el autor ha querido ofrecer su aporte intelectual al proceso catastral colombiano al concebirlo en el documento "Actualización de la Metodología de zonas Homogéneas Físicas Urbanas" y verlo hecho realidad en el proyecto piloto de la ciudad de Zipaquirá (Cundinamarca).

\section{La propuesta metodológica}

El objetivo central de este proyecto está orientado a la actualización de la metodología para la determinación de zonas homogéneas físicas urbanas, tomando como base la información proveniente de los planes de ordenamiento territorial municipal, la base teórica para su realización fue tomada de la investigación del autor mencionado anteriormente, en la cual se plantea la determinación de las zonas homogéneas físicas urbanas a través de la inclusión de dos nuevas variables, las cuales son tomadas de los planes de ordenamiento territorial. En definitiva son siete (7) las variables que conforman las zonas homogéneas físicas propuestas.

Las nuevas variables incluidas en la metodología son las siguientes:

1. Áreas de actividad

2. Tratamientos urbanísticos

La información correspondiente a cada una de estas variables es tomada directamente de los planes de ordenamiento territorial del municipio. Estas variables son la base fundamental para iniciar el estudio de las zonas homogéneas físicas ya que aportan los dos primeros dígitos en la determinación de la zonificación física urbana.

Las restantes cinco variables son las utilizadas normalmente en la metodología que tiene establecida Catastro para la determinación de zonas homogéneas físicas urbanas, en la cual se contempla la utilización de las siguientes variables.

\section{Destino económico \\ 4. Tipificación \\ 5. Servicios públicos \\ 6. Vías \\ 7. Topografía}

Con la determinación de la zonificación urbana mediante la utilización de la información proveniente de los planes de ordenamiento territorial se pretende, determinar una zonificación física, lo más cercana posible a la realidad urbana del municipio y que permita un cálculo más justo de los avalúos catastrales para los respectivos predios y por ende para sus propietarios. 


\section{Análisis de las variables para la determinación de zonas homogéneas físicas urbanas de zipaquirá}

Como se mencionó anteriormente las zonas homogéneas físicas urbanas son aquellos espacios geográficos dentro del perímetro urbano con características similares en cuanto a comportamiento de determinadas variables, las cuales serán estudiadas individualmente, para luego realizar el análisis conjunto para determinar las zonas homogéneas físicas finales.

\section{Áreas de actividad (Primera Variable)}

Son las divisiones del área urbana para el efecto de asignación de usos y demás reglamentaciones urbanísticas. Esta se refiere al régimen legal sobre el uso del espacio urbano. Comprende el conjunto de medidas y disposiciones generales que regulan el uso del suelo en el territorio.

Las áreas de actividad se dividen en áreas de actividad múltiple, áreas de actividad especializada y en áreas de futuros desarrollos.

Áreas de Actividad Múltiple: Son aquellas que por su localización estratégica dentro de la ciudad por sus características adquiridas a través del proceso de formación y consolidación de sus estructuras, constituyen sectores de atracción de varias actividades por lo tanto muestran tendencias a la mezcla de usos y a la intensificación de alguno de ellos.

Áreas de Actividad Especializada: Son aquellas que presentan un uso predominante que las caracterizan y a partir del cual se establecen la reglamentación y restricciones de los otros usos permitidos en el área, se pueden clasificar de acuerdo a su uso en:

- Áreas comerciales

- Áreas residenciales

- Áreas industriales

- Áreas institucionales o dotacionales

Áreas de Futuros Desarrollos: Son zonas que en la actualidad no tienen una utilización de actividades urbanas y son reserva para el proceso de urbanización, por lo general son los grandes lotes dentro del perímetro urbano y la zona de expansión urbana.

Las áreas de actividad es la primera variable que se estudia en la nueva metodología para la determinación de zonas homogéneas físicas, la información concerniente a esta variable se tomó directamente del plan de ordenamiento territorial del municipio de Zipaquirá (Acuerdo No. 12 de 2000).

El análisis de las áreas de actividad en el plan de ordenamiento territorial del municipio de Zipaquirá es abordado a través de las denominadas áreas morfológicas homogéneas, las cuales se caracterizan porque constituyen sectores diferenciados de desarrollo dentro del

116 Jaime Silva Herrera 
municipio, y valoran cualitativamente el territorio. Sirven para caracterizar las condiciones y potencialidades urbanísticas que éste posee, así como para orientar las intervenciones que se deberán realizar, es decir se constituyen en divisiones establecidas en el área urbana con el fin de asignarles usos y reglamentaciones urbanísticas específicas.

Teniendo como base las áreas morfológicas homogéneas suministradas por el POT del municipio y la vocación y caracterización de cada una de ellas, se determinaron las áreas de actividad, las cuales van a ser utilizadas en la determinación de las zonas homogéneas físicas en el área urbana del municipio.

Como resultado de este análisis el POT determinó las siguientes áreas de actividad para el municipio de Zipaquirá.

\begin{tabular}{|c|l|}
\hline CóDIGo & \multicolumn{1}{|c|}{ ÁREADE ACTIVIDAD } \\
\hline 1 & MÚLTIPLE \\
\hline 2 & RESIDENCIAL GENERAL \\
\hline 3 & RESIDENCIALESPECIAL \\
\hline 4 & TURÍSTICAY CULTURAL \\
\hline 5 & $\begin{array}{l}\text { ADMINISTRATIVAY } \\
\text { CULTURAL }\end{array}$ \\
\hline 6 & INDUSTRIALY SERVICIOS \\
\hline
\end{tabular}

Estas áreas de actividad se constituyen en la primera variable estudiada en la determinación de los estudios de zonas homogéneas físicas a través de la nueva metodología, a cada área de actividad se le asignó un código, tal cual aparece en el cuadro anterior, dicho número se constituye en el primer dígito de las zonas homogéneas físicas propuestas (Mapa No. 1).

\section{Tratamientos urbanísticos (Segunda Variable)}

Se entiende por tratamiento urbanístico el mecanismo normativo para el manejo del territorio clasificado como suelo urbano y de expansión urbana, de acuerdo con sus características urbanas para asegurar un desarrollo armónico y ordenado de las actuaciones urbanísticas.

Estos definen áreas con manejo diferenciado de los usos y normas para cada una de las áreas de actividad, estas pueden encontrarse en diferentes áreas de actividad y a su vez un área de actividad puede contener una o varias áreas de tratamiento. Ésta determina y direcciona las actuaciones urbanísticas permitidas para una zona específica de la ciudad teniendo en cuenta el uso del suelo.

Los tratamientos urbanísticos constituyen la segunda variable incluida en la nueva metodología para la determinación de zonas homogéneas físicas urbanas, al igual que la anterior la fuente de información principal para su estudio es el POT del municipio, en el cual se encuentran estipulados los tratamientos urbanísticos para cada zona del municipio. 
El plan de ordenamiento territorial estableció para el núcleo urbano del municipio de Zipaquirá los siguientes tratamientos urbanísticos:

\begin{tabular}{|c|l|}
\hline CÓDIGO & $\begin{array}{l}\text { TRATAMIENTO } \\
\text { URBANÍSTICO }\end{array}$ \\
\hline 1 & $\begin{array}{l}\text { CONSERVACIÓN } \\
\text { URBANÍSTICA }\end{array}$ \\
\hline 2 & $\begin{array}{l}\text { CONSOLIDACIÓN } \\
\text { URBANÍSTICA }\end{array}$ \\
\hline 3 & $\begin{array}{l}\text { RENOVACIÓN } \\
\text { URBANÍSTICA }\end{array}$ \\
\hline 4 & $\begin{array}{l}\text { MEJORAMIENTO } \\
\text { INTEGRAL }\end{array}$ \\
\hline 5 & DESARROLLO \\
\hline 6 & RENOVACIÓN ESPECIAL \\
\hline
\end{tabular}

\section{Tratamiento de consolidación urbanística}

Es el mecanismo normativo aplicable a los predios urbanizados que se encuentran ubicados en suelos clasificados como urbano que requieren actuaciones urbanísticas que permitan afianzar las características urbanas del sector.

Se aplica para zonas que contienen edificaciones que presentan características volumétricas homogéneas, requiriendo que las nuevas actuaciones urbanísticas de construcción se realicen en condiciones análogas a las existentes en el sector, con el fin de consolidar la forma urbana existente (Acuerdo $12 \mathrm{de}$ 2000, artículo 63).

\section{Tratamiento de renovación urbana}

Es el aplicable a sectores ubicados dentro del suelo urbano, que requieren introducir modificaciones sustanciales al uso de la tierra y de la construcción para detener procesos de deterioro físico y ambiental, con el fin de obtener un aprovechamiento intensivo de la infraestructura establecida de servicios, la descongestión del tráfico urbano, la rehabilitación de bienes históricos y culturales, la utilización más eficiente de inmuebles históricos y culturales, la utilización más eficiente de inmuebles urbanos para beneficio de la comunidad. (Acuerdo 12 de 2000, artículo 65).

\section{Tratamiento de mejoramiento integral}

Es el mecanismo normativo aplicable a sectores catalogados como de desarrollo incompleto que requieren de acciones de habilitación y mejoramiento del entorno, los cuales incluyen la previsión y dotación de espacios requeridos para el uso público y equipamiento comunal, infraestructura vial y de servicios públicos $\mathrm{y}$, en general, requieren de obras que garanticen un mejoramiento urbano del territorio y de las viviendas. Para mejorar el desarrollo urbanístico de estas áreas se requiere la elaboración de planes parciales (Acuerdo 12 de 2000, artículo 64).

\section{Tratamiento de desarrollo}

Es el mecanismo normativo aplicable a los predios rústicos (sin urbanizar) que se 
encuentran ubicados en suelos clasificados como urbano y de expansión urbana, que requieren de actuaciones urbanísticas de urbanización, para desarrollarse en usos urbanos. Para el desarrollo urbanístico de estas áreas se requiere la elaboración de planes parciales (Acuerdo 12 de 2000, artículo 62).

Para los tratamientos urbanísticos correspondientes a conservación urbanística y renovación especial, no existe dentro del Acuerdo 12 de 2000, ninguna definición específica.

A cada tratamiento urbanístico se le asignó un código, dicho número se constituye en el segundo dígito de las zonas homogéneas físicas propuestas (Mapa No. 2).
Como síntesis se puede afirmar que las áreas de actividad y los tratamientos urbanísticos constituyen el elemento normativo de las zonas homogéneas físicas, debido a que estas áreas se encuentran bajo reglamentación determinada por el plan de ordenamiento territorial, los cual constituye un aporte fundamental para determinar una zonificación urbana acorde a los lineamientos de uso y ocupación del territorio determinados por el POT.

Según lo establecido en el plan de ordenamiento territorial del municipio de Zipaquirá las áreas de actividad se encuentran reglamentadas con determinados tratamientos urbanísticos los cuales se denotan en el siguiente cuadro:

\begin{tabular}{|r|l|l|}
\hline \multicolumn{2}{|c|}{ ÁREASDE ACTIVIDAD } & TRATAMIENTOS URBANISTICOS \\
\hline 1 & MÚLTIPLE & - CONSOLIDACIÓN URBANÍSTICA \\
& & - RENOVACIÓN URBANA \\
& & - DESARROLLO \\
\hline 2 & RESIDENCIAL GENERAL & - CONSOLIDACIÓN URBANÍSTICA \\
& & - DESARROLLO \\
\hline 3 & RESIDENCIALESPECIAL & - CONSOLIDACIÓNURBANÍSTICA \\
& & - DESARROLLO \\
\hline 4 & TURÍSTICAYCULTURAL & - CONSERVACIÓNURBANÍSTICA \\
& & - RENOVACIÓNURBANA \\
\hline 5 & ADMINISTRATIVAYCULTURAL & - CONSOLIDACIÓN URBANISTICA \\
& & - CONSERVACIÓN \\
\hline 6 & INDUSTRIALYSERVICIOS & - CONSOLIDACIÓNURBANISTICA \\
& & - DESARROLLO \\
\hline
\end{tabular}


Aquí concluye lo que podríamos denominar como una labor eminentemente de oficina, es decir la lectura e interpretación de cada una de las áreas de actividad y tratamientos urbanísticos, estipulados tanto en el articulado del POT como en los respectivos mapas.

Enseguida entraremos a observar las otras cinco variables que obligatoriamente deben ser capturadas en terreno.

\section{Destinación económica (Tercera Variable)}

Es la actividad económica predominante en la zona. Dentro de esta variable se tiene en cuenta, específicamente para la zona urbana del municipio de Zipaquirá los siguientes destinos económicos: Residencial, comercial, industrial, dotacional y predios no edificados.

Zona Residencial: Es la zona o espacio cuyas edificaciones tienen como función arquitectónica y ambiental principal de dar albergue de forma adecuada a personas.

Zona Comercial: Es el espacio cuya edificación está dedicada a la compra/ venta de bienes y servicios.

Zona Industrial: Es el espacio urbano en cuyas edificaciones se desarrollan actividades de explotación, transformación o ensamblaje de materias primas, que utilizan como soporte de la actividad industrial áreas urbanas desarrolladas y edificaciones con características, servicios, y especificaciones apropiadas para la industria.
Cuando la industria se lleva a cabo en urbanizaciones o ciudades específicamente previstas para industria, se denominará complejo industrial, el cual sólo podrá ser autorizado dentro de los límites de las zonas industriales.

Según la actividad predominante y para efectos de sus manejos, los usos industriales pueden corresponder a industria transformadora o a industria extractiva.

Zona Dotacional: Son aquellos usos urbanos cuya función es la de prestar los diferentes servicios requeridos como soporte de las actividades de la población.

Zona de Predios no Edificados: Son lotes que no han tenido desarrollo por construcción, es decir, que no se han desarrollado las acciones encaminadas a la construcción de edificios, casas, etc., y que se encuentran dentro de los perímetros urbanos y de expansión.

El uso del suelo o destinación económica es una de las variables que se utiliza en la metodología tradicional de la Subdirección de Catastro a nivel nacional, la información de esta variable se captura directamente en terreno, mediante inspección ocular a cada una de las zonas determinadas anteriormente mediante la superposición de las áreas de actividad y los tratamientos urbanísticos.

La información que se capturó en terreno tuvo como base las clases de uso definidas en dicha clasificación y se utilizó como unidad de estudio los polígonos obtenidos mediante la superposición de las variables 
áreas de actividad y tratamientos urbanísticos, es decir se analizó la destinación económica para cada tipo de polígono con áreas de actividad y tratamiento urbanístico.

A cada tipo de destinación económica se le asignó un código, tal cual aparece en el siguiente cuadro, dicho número se constituye en el tercer dígito de las zonas homogéneas físicas propuestas (Mapa No. 3).

\begin{tabular}{|c|l|}
\hline CÓDIGO & \multicolumn{1}{c|}{ USO } \\
\hline 1 & RESIDENCIAL \\
\hline 2 & COMERCIAL \\
\hline 3 & INDUSTRIAL \\
\hline 4 & DOTACIONAL \\
\hline 5 & PREDIONOEDIFICADO \\
\hline
\end{tabular}

\section{Tipo según destinación económica (Cuarta Variable)}

Esta variable es una subdivisión de la variable anterior, para zonas urbanas construidas la subdivisión se da según el tipo de construcción en que se desarrolla la actividad económica.

\section{Tipo en zona residencial}

Corresponde a la tipificación de la vivienda, el cual define el tipo predominante en cada zona, con un puntaje determinado dentro de un rango llamado tipo y que tiene sus aproximaciones pero también sus diferencias con el denominado estrato socioeconómico.
De acuerdo con las características de las edificaciones en las cuales se desarrolla $\mathrm{y}$ el régimen de propiedad de las mismas se identificaron las siguientes modalidades de uso residencial, para efectos de su reglamentación:

- Vivienda unifamiliar

- Vivienda bifamiliar

- Vivienda multifamiliar

- Agrupaciones o conjuntos

- Vivienda compartida

Vivienda unifamiliar: Corresponde al desarrollo residencial en el cual un lote de terreno está ocupado por una unidad predial destinada a dicho uso y que no comparte con los demás inmuebles de la zona ningún tipo de área o servicio comunal de carácter privado.

Vivienda bifamiliar y trifamiliar: Corresponde al desarrollo residencial en el cual un lote de terreno está ocupado por dos o tres unidades prediales que comparten en común y proindiviso la propiedad del terreno, así como elementos de la edificación y áreas de acceso y aislamientos y cuentan con reglamentos de propiedad horizontal o de copropiedad.

Vivienda multifamiliar: Corresponde a edificaciones desarrolladas sobre un lote de terreno que comprende más de tres unidades prediales independientes, generalmente en edificios de varios pisos.

Este tipo de desarrollo prevé áreas y servicios comunales dentro de los edificios, cuya propiedad y utilización privadas se definen en reglamentos de propiedad horizontal. 
Agrupaciones o conjuntos: Son desarrollos residenciales conformados por varias edificaciones constitutivas de unidades de vivienda unifamiliar, bifamiliar o multifamiliar, que comparten, además de las estructuras o áreas comunales privadas de cada edificación, o sea las de su propio régimen de comunidad, zonas y servicios comunes a toda la agrupación o conjunto, como vías privadas, zonas de estacionamiento, zonas verdes, muros de cerramiento, porterías, etc.

Vivienda compartida: Está constituida por edificaciones que albergan espacios aptos como unidades de vivienda, que no están segregados predialmente ni sometidos a régimen de propiedad horizontal o de copropiedad, a pesar de compartir áreas y servicios.

La tipificación de la vivienda se da para todas las modalidades de uso residencial vistas anteriormente y se clasifican de la siguiente manera:

\begin{tabular}{|c|l|c|}
\hline CÓDIGO & TIPIFICACIÓN & PUNTAJE \\
\hline 1 & BAJO-BAJO & $0-10$ \\
\hline 2 & BAJO & $11-28$ \\
\hline 3 & MEDIO-BAJO & $29-46$ \\
\hline 4 & MEDIO & $47-64$ \\
\hline 5 & MEDIO-ALTO & $65-82$ \\
\hline 6 & ALTO & $83-100$ \\
\hline
\end{tabular}

Tipo en Zona Comercial: De acuerdo con su cobertura territorial y para efectos de establecer sus características generales e impacto y definir criterios de manejo, se distinguen tres tipos de comercio así:
- Cobertura local

- Cobertura zonal

- Cobertura Metropolitana

\section{Cobertura Local}

Corresponde a la venta de bienes y servicios de consumo doméstico requeridos por la comunidad de residentes de un sector.

Presenta dos modalidades principales de desarrollo: en áreas o espacios de edificaciones residenciales que fueron destinadas al uso comercial sin adecuaciones físicas importantes, ni del espacio mismo, ni del resto de la vivienda que se mantiene como tal; o en establecimientos situados en edificaciones multifamiliares o en manzanas o en centros comerciales de las urbanizaciones.

Presenta las siguientes características principales, entre otras:

- Venta de bienes de primera necesidad que no requiere zonas especiales de exhibición y bodegaje, y venta de servicios de primera necesidad.

- Abastecimiento mediante vehículos pequeños o medianos que no requieren zonas especializadas para cargue y descargue ni vías de acceso de gran magnitud.

- Poca o ninguna generación de empleo

- Los usuarios acceden a pie por lo general y su permanencia en el estable- 
cimiento suele ser corta. Por lo tanto no se advierten mayores exigencias de establecimiento de vehículos automotores.

- Tiene horarios laborales y de atención al público diurno, sin influencia concentrada en horas, días o temporadas.

- No requiere servicios de infraestructura especiales ni adicionales a los previstos para una zona residencial.

- No requiere gran especialización de las edificaciones ni del espacio público en el cual se ubican.

- No requiere ni genera usos complementarios

Por sus características generales se considera un comercio bajo impacto, que por lo tanto puede aparecer mezclado con cualquier tipo de uso principal.

Predios comerciales locales: Se consideran predios comerciales locales los que tengan destinado al comercio un área igual o menor a cien (100) metros cuadrados, incluidos los anexos necesarios tales como cuartos de depósito, en los cuales se vendan bienes o servicios de consumo doméstico requeridos por la comunidad de residentes de un sector de la ciudad, que no necesiten de adecuaciones especiales de bodegaje ni de infraestructuras especiales diferentes a las utilizadas para la corriente exhibición de la mercancía, como por ejemplo:
- Venta de artículos y comestibles de primera necesidad, tales como tiendas de barrio, fruterías, panaderías, productos lácteos, expendio de carnes y pescado, salsamentaria, bebidas no alcohólicas, y similares, siempre y cuando no sean supermercados.

- Venta de artículos varios de consumo doméstico tales como droguerías, cigarrerías y papelerías.

- Servicios personales tales como sastrerías, modisterías, peluquerías, salón de belleza, agencias de lavandería y tintorerías, remontadoras de calzado y similares.

- Servicios alimenticios al detal, tales como cafeterías y similares.

\section{Cobertura zonal}

Comprende los usos comerciales de intercambio de bienes y servicios que suplen demandas especializadas generadas en la comunidad de una zona urbana.

Incluye gran diversidad de usos comerciales que se presentan generalmente en las siguientes formas:

- En adecuaciones totales o parciales de edificaciones originalmente residenciales.

- En establecimientos especializados para el uso comercial ubicados en primeros pisos de edificaciones residenciales o en manzanas o centros comerciales de las 
urbanizaciones y en edificaciones especializadas para el uso comercial con venta de bienes en los primeros pisos y venta de servicios en los pisos superiores.

Presenta las siguientes características principales, entre otras:

- Venta especializada de bienes al detal que pueden requerir vitrinas de exhibición y zonas de bodegaje.

- Actividades mercantiles de venta de servicios

- Generación mediana de empleo

- Requiere de zonas de estacionamiento para empleados y usuarios que acceden en una gran proporción en forma vehicular y tiene una permanencia media o prolongada en los establecimientos.

- Funciona en horarios laborales diurnos y nocturnos con posible afluencia concentrada de usuarios en horas, días y temporadas determinadas.

- Puede requerir servicios de infraestructura específicos, especialmente de telefonía en el caso de las actividades mercantiles y profesionales de servicios.

- Genera usos complementarios, por lo cual propicia la formación apulatina de ejes o sectores de actividad comercial.

- Con excepciones, se considera de bajo impacto ambiental y social, pero de notorio impacto urbano causado por el tránsito y congestión de personas, vehículos y actividades a que da lugar, especialmente cuando se presenta en adecuaciones que no resuelven satisfactoriamente dicho impacto.

Predios comerciales zonales: Se consideran predios comerciales zonales aquellos que ocupen un área superior a cien (100) metros cuadrados.

También son predios comerciales zonales los destinados a la venta de bienes o servicios que suplen demandas especializadas diferentes al consumo doméstico generadas en la comunidad de una zona de la ciudad, como por ejemplo almacenes de artículos de ropa, repuestos, videotiendas, exhibición y venta de vehículos, restaurantes, servitecas, licoreras, etc.

En todo caso son predios comerciales zonales en razón a su impacto o especialidad de edificaciones los destinados a:

- La venta de alimentos y bebidas en supermercados y similares, con áreas como parqueo, bodegaje, cuartos fríos, etc.

- La venta de bienes o servicios para automóviles y autopartes.

También son predios comerciales zonales las oficinas, entendiendo por tales los predios destinados a la prestación de servicios profesionales, técnicos especiales o administrativos. 


\section{Cobertura Metropolitana}

Comprende los usos comerciales orientados a satisfacer demandas de bienes y servicios generados en toda la ciudad mediante la oferta concentrada de gran variedad de bienes.

Este comercio se presenta en dos modalidades:

- Como agrupación de establecimientos de venta de bienes y servicios que conforman unidades arquitectónicas y urbanísticas y comparten zonas y servicios comunales (centros comerciales, empresariales, etc.).

- En establecimientos únicos con oferta diversificada de bienes (almacenes de departamentos, centros de exposición, etc.). Estos establecimientos pueden formar o no parte de los anteriores.

Presentan las siguientes características principales:

- Se presentan en edificaciones o agrupaciones de gran magnitud especialmente diseñadas para tal fin.

- Prestan servicios en horarios laborales especiales con influencia concentrada en horas, días y temporadas determinadas.

- Constituyen centros de empleo con afluencia y permanencia muy altas de trabajadores. El acceso, tanto de éstos como de los usuarios se efectúa en forma vehicular, ya sea mediante la utilización de transporte público o privado.
Requieren por lo tanto estar ubicados sobre vías del plan vial arterial y contar con amplias zonas de estacionamiento.

- Se abastecen mediante vehículos medianos o pesados para lo cual requieren zonas especializadas de cargue y descargue.

- Debido a la permanencia de usuarios y empleados en los establecimientos, requieren servicios complementarios como parte de la edificación o conjunto comercial.

Por sus características físicas y funcionales se considera un tipo de comercio de alto impacto urbanístico, que requiere soluciones particulares para cada caso en cual se proponga su desarrollo.

Predios comerciales metropolitanos: Se consideran predios comerciales metropolitanos:

- Aquellos que por su ubicación o magnitud suplen las demandas de bienes y servicios en general o aquellos que por razón de su impacto, volumen o su inconveniente de funcionamiento requieren de localización especial.

Son predios comerciales metropolitanos:

- Aquellos en los que se realice la oferta de bienes o servicios en edificaciones especializadas o complejos arquitectónicos cuya área sea mayor o igual a 20.000 metros cuadrados de construcción. 
- Aquellos en los que se realice la oferta concentrada de gran variedad de bienes o servicios en edificaciones especializadas como almacenes por departamentos, oficinas o locales en centros o complejos empresariales.

- Aquellos ubicados en centros comerciales

- Los centros comerciales

- Los hoteles

- Aquellos predios comerciales ubicados en zonas de comercio mayoritario, es decir donde hay una agrupación de establecimientos en una o varias cuadras.

- Las zonas de comercio especializado en sectores de la ciudad, tales como zonas de venta de repuestos automotrices, zonas de ferreteros, zonas de talleres de reparación automotriz, sanandresitos, zonas de venta de materiales para construcción, y las análogas a las anteriores.

En todo caso son predios comerciales metropolitanos los que se destinen a:

- Griles y similares

- Galleras

- Campos de tejo

- Moteles y similares

- Almacenamiento en grandes depósitos o bodegas y similares

- Servicios funerarios

- Y los análogos a los anteriores
Igualmente serán metropolitanos los predios comerciales que se desarrollen en áreas superiores a 20.000 metros cuadrados.

Los diferentes tipos del destino comercial se pueden encontrar de la siguiente manera:

TIPIFICACIÓN EN DESTINO COMERCIAL

\begin{tabular}{|c|l|}
\hline CóDIGO & TIPIFICACIÓN \\
\hline 1 & LOCAL \\
\hline 2 & ZONAL \\
\hline 3 & METROPOLITANO \\
\hline
\end{tabular}

Tipo en Zona Industrial: La industria se divide en cuatro tipos, de acuerdo con el impacto ambiental y urbanístico que genera la actividad industrial, así:

Industria transformadora Tipo 1: Es aquella industria considerada compatible con otros usos en razón de su bajo impacto ambiental y urbanístico.

Se ubica en espacios que forman parte de edificaciones comerciales o de vivienda unifamiliar o en locales o bodegas independientes.

Presenta las siguientes características principales:

- Manufactura artesanal de productos, generalmente manual o con equipos caseros. 
- Abastecimiento de materias primas y transporte de productos terminados mediante vehículos livianos.

- Poca generación de empleo

- Funcionamiento en horarios laborales preferiblemente diurnos

- No requiere servicios de infraestructura especiales ni produce ruidos, olores, ni efluentes contaminantes.

Industria transformadora Tipo 2: Es aquella industria compatible con otros usos dado su bajo impacto ambiental, pero con restricciones de localización debido a su magnitud y alto impacto urbanístico.

Se ubica en bodegas o edificaciones especializadas dentro de zonas y complejos industriales.

Presenta las siguientes características principales:

- Manufactura, producción o transformación de productos, mediante técnicas, equipos y laboratorios especializados, que pueden requerir formas específicas de prestación de servicios públicos e infraestructura de los mismos.

- Abastecimiento de materias primas y transporte de productos terminados mediante vehículos medianos o pesados, por lo cual requiere zonas de cargue y descargue y un sistema vial adecuado.

- Generación de empleo masivo y mano de obra especializada, por lo cual requiere zonas de estacionamiento para trabajadores y posibilidad de acceso por transporte público.

- Fomenta la aparición de establecimientos a usos comerciales complementarios de la industria, como los "puntos de venta", restaurantes, establecimientos bancarios, etc.

Industria Transformadora 3: Es aquella industria que tiene restricciones de localización debido a su alto impacto ambiental, a su magnitud considerable y a su alto impacto urbanístico.

Requiere para su funcionamiento, además de edificaciones especializadas, elementos o equipos para el control de contaminación por ruido, olores, vapores o efluentes líquidos, así como un espacio público con características especiales que permita la accesibilidad de vehículos pesados y prevea franjas de control ambiental o de aislamiento apropiadas.

Requiere formas específicas de prestación de servicios públicos e infraestructura de los mismos, tanto para el funcionamiento de la actividad industrial, como para el control de los efluentes contaminantes que produce.

Debido a su alto impacto se trata de un uso compatible en las zonas industriales y prohibido en las demás áreas de actividad y zonas.

\section{Industria extractiva}

Es aquella cuya actividad principal consiste en la explotación y tratamiento 
de materiales rocosos, arcillosos, arenosos y en general, de los demás recursos naturales procedentes de minas subterráneas y superficiales, canteras y pozos.

Incluye además todas las actividades suplementarias para manejo, y beneficio de minerales y otros materiales crudos, tales como triturado, cribado, lavado, clasificación y demás preparaciones necesarias para entregar el material en el mercado.

La industria extractiva no es un uso urbano. En consecuencia se considera un uso prohibido dentro de las áreas urbanas.

La síntesis de los tipos de industrias que pueden estar ubicados en las zona urbana de un municipio se muestra a continuación:

\section{TIPIFICACIÓN EN DESTINO INDUSTRIAL}

\begin{tabular}{|c|l|}
\hline CÓDIGO & \multicolumn{1}{|c|}{ TIPIFICACIÓN } \\
\hline 1 & Transformadora Tipo 1 \\
2 & Transformadora Tipo 2 \\
3 & Transformadora Tipo 3 \\
4 & Extractiva \\
\hline
\end{tabular}

TIPO EN ZONA DOTACIONAL: de acuerdo a sus características los tipos en zona dotacional se clasifican de la siguiente manera:

Asistenciales: corresponden a aquellos destinados a la satisfacción de las necesidades básicas sociales de salud, asistencia y bienestar social, tales como Centros comunales, centros de salud, clínicas, hospitales, guarderías, ancianatos, entre otros.

Educativos: corresponden a aquellos destinados a la satisfacción de las necesidades de escolaridad de la población, tales como jardines infantiles, escuelas, colegios, centros de educación superior, institutos de capacitación técnica, universidades, entre otros.

Culturales: Corresponden a aquellos destinados a la satisfacción de necesidades y actividades relacionadas con el cultivo del intelecto y del espíritu artístico de los habitantes, tales como bibliotecas, salas de teatro, museos, galerías de arte, centros culturales, auditorios, entre otros.

Administrativos y de seguridad: Corresponden a aquellos destinados a la prestación de servicios relacionados con la administración, gobierno y seguridad ciudadana, tales como, sedes de entidades gubernamentales, notarías, estaciones y subestaciones de policía, cuarteles, instalaciones militares, cárceles, estaciones de bomberos, entre otros.

Recreativos y Deportivos: Corresponden a aquellos destinados a la práctica del ejercicio físico como actividad de recreo $u$ oficio $y$ demás necesidades de recreación y deporte, tales como polideportivos, conchas acústicas, entre otros.

Religiosos y de Culto: Corresponden a aquellos destinados a la prestación de 
servicios religiosos y de culto, tales como iglesias, capillas, conventos, seminarios, sedes de diferentes cultos, entre otros.

De Abastecimiento: corresponden a aquellos destinados a la prestación de servicios de soporte a la economía de la ciudad, tales como plazas de mercado, centros de abasto, centros de acopio, entre otros.

Servicios Urbanos Especiales: Corresponden a aquellos destinados a la prestación de servicios y dotaciones urbanas que satisfacen necesidades especiales, tales como cementerios, terminales de transporte, aeropuertos entre otros.

El resumen de los tipos dotacionales los podremos observar en el siguiente cuadro:

\section{TIPIFICACIÓN EN DESTINO DOTACIONAL}

\begin{tabular}{|c|l|}
\hline CóDIGO & TIPIFICACIÓN \\
\hline 1 & ASISTENCIALES \\
2 & EDUCATIVOS \\
3 & CULTURALES \\
4 & ADMINISTRATIVOS Y \\
& DE SEGURIDAD \\
5 & RECREATIVOS Y \\
& DEPORTIVOS \\
6 & DE CULTO \\
7 & DE ABASTECIMIENTO \\
8 & SERVICIOS URBANOS \\
& ESPECIALES \\
\hline
\end{tabular}

Tipo en destino Predios no Edificados: Para esta zona la tipificación se efectúa teniendo en cuenta la condición de urbanizable o no que presenten los predios que la conforman, así:

Urbanizables no urbanizados; son aquellos predios que a pesar de ser aptos para el desarrollo como urbanización, no han sido desarrollados como tales.

No urbanizables; son predios que presentan alguna característica especial por la cual no pueden ser urbanizados.

Urbanizados no edificados; corresponde a aquellos lotes que a pesar de encontrarse completamente urbanizados no se ha iniciado completamente el proceso de construcción.

El siguiente es el resumen de este tipo de destino:

\section{TIPIFICACIÓN EN DESTINO PREDIO NO EDIFICADO}

\begin{tabular}{|c|l|}
\hline CÓDIGO & \multicolumn{1}{|c|}{ TIPIFICACIÓN } \\
\hline 1 & URBANIZABLE NO \\
\hline & URBANIZADO \\
\hline 2 & NO URBANIZABLE \\
\hline 3 & $\begin{array}{l}\text { URBANIZADO NO } \\
\text { EDIFICADO }\end{array}$ \\
\hline
\end{tabular}

Recordemos que el tipo según destinación económica, es la cuarta variable de la metodología propuesta, por lo tanto, cualquiera que fuese el tipo de destino hallado en la zona, este llevará su respectivo código, que a su vez se convierte en el cuarto dígito de la zona Homogénea Física propuesta (Mapa No. 4). 


\section{Servicios públicos (Quinta Variable)}

El estudio de esta variable se realiza de la misma forma que en la metodología actual de la Subdirección de Catastro. En esta variable se estudia la cobertura de servicios públicos en la zona, la información es capturada directamente en terreno, se verifica la existencia o no de servicios públicos domiciliarios para cada zona. Para su calificación se tiene en cuenta la siguiente:

Sin servicios: Zonas que no cuentan con uno o dos servicios públicos.

Básicos Incompletos: Zonas que cuentan con uno o dos servicios públicos.

Básicos: Zonas que cuentan con servicios de Energía, Acueducto y Alcantarillado.

Básicos más Complementarios: Zonas que poseen todos los servicios básicos más otro nivel adicional.

El resumen de esta variable se presenta en el siguiente cuadro:

\begin{tabular}{|c|l|}
\hline CÓDIGO & \multicolumn{1}{|c|}{ CATEGORÍA } \\
\hline 1 & SIN SERVICIOS \\
2 & BÁSICOS INCOMPLETOS \\
3 & BÁSICOS \\
4 & BÁSICOS MÁS \\
& COMPLEMENTARIOS \\
\hline
\end{tabular}

Como Se puede observar, a cada categoría de servicios público se le asignó un código, tal cual aparece en el cuadro anterior; dicho número se constituye en el quinto dígito de las zonas homogéneas físicas propuestas (Mapa No. 5).

\section{Vías (Sexta Variable)}

Es la cobertura o no que tiene determinada zona de la cabecera municipal y de la zona de expansión en cuanto a su acceso vial y al estado y condiciones de ésta. Se clasifican de la siguiente manera:

Pavimentadas: Son vías que poseen su capa de rodamiento en asfalto, cemento, ladrillo o adoquines.

No pavimentadas: Son las vías que se encuentran recebadas o en afirmado o únicamente con descapote.

Peatonales: Vías que solo permiten la circulación de peatones.

Sin vías: Carecen de vías.

La calificación se hace mediante verificación en campo tomando como base el siguiente cuadro:

\begin{tabular}{|c|l|}
\hline CÓDIGO & \multicolumn{1}{|c|}{ CATEGORÍA } \\
\hline 1 & PAVIMENTADA \\
2 & SIN PAVIMENTAR \\
3 & PEATONALES \\
4 & SIN VÍAS \\
\hline
\end{tabular}

Para cada zona delimitada por cualquiera de las categorías expuestas en el cuadro anterior, se le asigna su respectivo código, 
dicho número se constituye en el sexto dígito dentro de las zonas homogéneas físicas propuestas (Mapa No. 6).

\section{Topografía (Séptima Variable)}

En esta variable se estudian características naturales relacionadas con el relieve, para ello se tienen en cuenta factores como pendiente del terreno para clasificar las diferentes zonas, la información de esta variable es capturada directamente en terreno mediante la inspección ocular en la zona urbana. Para la calificación de esta variable se tiene en cuenta la siguiente clasificación:

\begin{tabular}{|c|l|}
\hline CÓDIGO & \multicolumn{1}{|c|}{ CATEGORÍA } \\
\hline 1 & PLANA $(0-7 \%)$ \\
2 & INCLINADA $(7-14 \%)$ \\
3 & EMPINADA $>14 \%$ \\
\hline
\end{tabular}

Para cada zona delimitada por cualquiera de las categorías expuestas en el cuadro anterior, se les asigna un código, este número proporciona el séptimo y último dígito para determinar las zonas homogéneas físicas propuestas (Mapa No. 7).

Hasta aquí se ha desarrollado y descrito cada una de las siete variables que componen los estudios de zonas homogéneas físicas urbanas propuestas, enseguida y para dar mayor ilustración sobre el proceso e interpretación, el autor ha desarrollado el cuadro "variables para la definición de zonas homogéneas físicas", que es la síntesis de todas y cada una de las variables propuestas, con sus correspondientes códigos y que sirve de gran ayuda sobre todo en las actividades de terreno. Apoyados en el anterior cuadro, se desarrollan dos ejemplos, veámoslos:

\section{Ejemplo 1:}

Zona Homogénea Física Propuesta No.

Área de actividad, múltiple (Residencial-comercial)

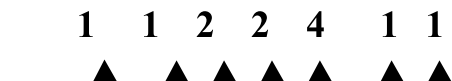

Tratamiento urbanístico, conservación

Destino económico, comercial

Tipo según destino económico comercial, zonal

Servicios, básicos más complementarios

Vías, pavimentadas

Topografía, plana 


\section{Ejemplo 2:}

Zona Homogénea física propuesta No.

Área de actividad, administrativa y cultural

Tratamiento urbanístico, desarrollo

Destinación económica, predio no edificado

Tipo según destino económico predio no edificado, urbanizable no

Urbanizado.

Servicios, básicos

Vías, sin pavimentar

Topografía, plana

\section{Zonas homogéneas físicas propuestas}

Después del análisis de cada una de las variables descritas anteriormente, tanto en oficina como en terreno, el proceso para la determinación de los estudios de zonas homogéneas físicas urbanas propuestas para Zipaquirá y en general para cualquier otra zona urbana es el siguiente:

1. Adquirir el acuerdo debidamente aprobado del P.O.T. del municipio de Zipaquirá (Acuerdo 12 de 2000).

2. Extractar del Acuerdo del POT 1o concerniente a las "Áreas de Actividad" incluyendo su respectivo plano (Mapa No. 1).
3. Extraer del Acuerdo del P.O.T., lo pertinente a los "tratamientos urbanísticos" incluyendo su respectivo plano (Mapa No. 2).

4. Pasar la información tanto de áreas de actividad como de tratamientos urbanísticos, a la cartografía IGAC de trabajo, aquí hay que tener mucho cuidado cuando las escalas son diferentes.

5. Determinar para cada área de actividad su correspondiente tratamiento urbanístico (labor de oficina).

6. Con los polígonos resultantes del paso 5, determinar para cada uno de ellos en terreno las variables, destino económico, tipo según destino económico, servicios, vías y topografía (Mapas No. 3, 4, 5, 6, 7). 


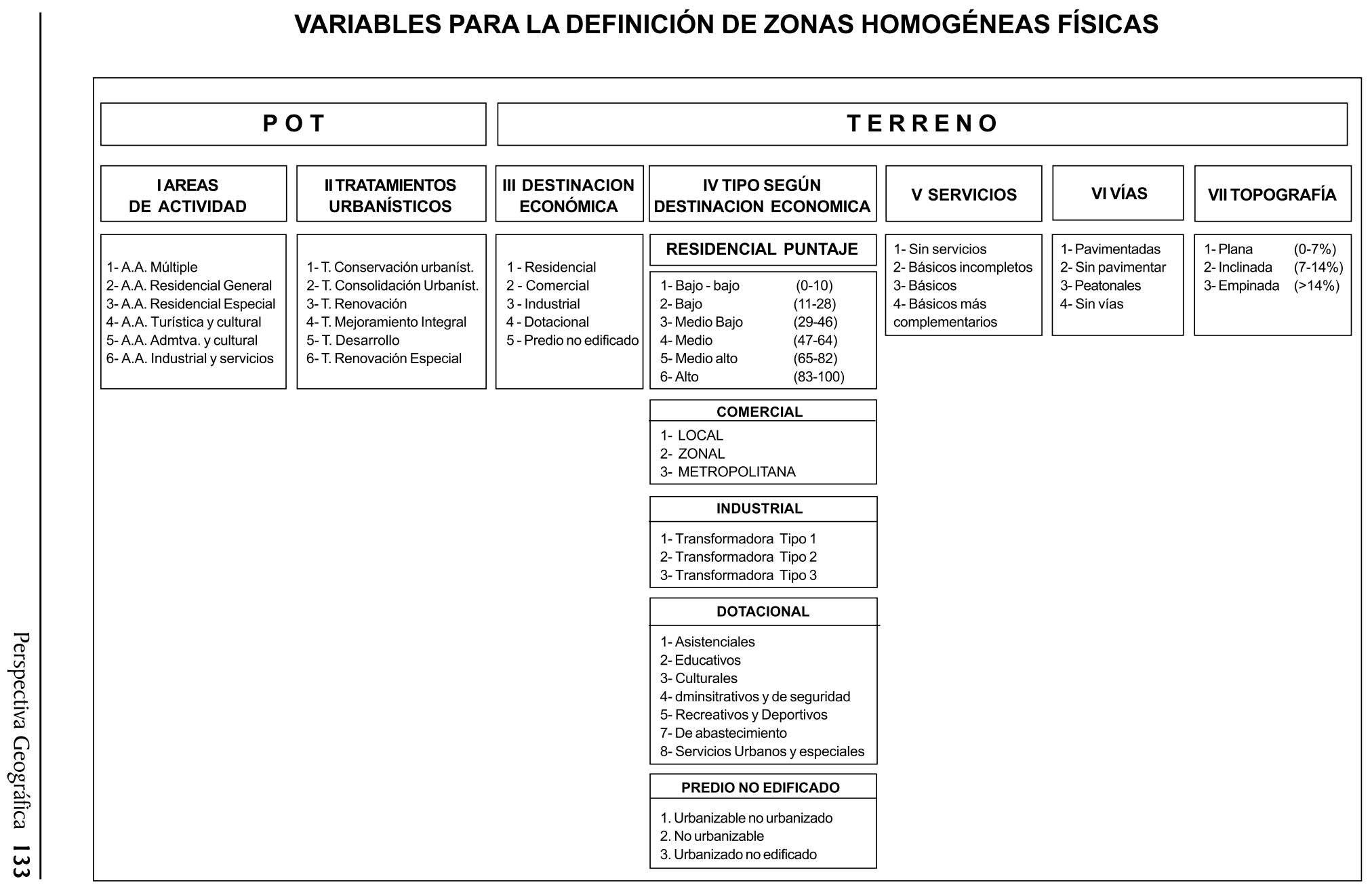


7. Haga un análisis integral del resultado obtenido, tenga en mente el proceso de homogenización más que el de individualización de variables.

8. Los nuevos polígonos obtenidos, fruto de los pasos 6 y 7, generan las zonas homogéneas físicas urbanas propuestas (Mapa de zonas homogéneas físicas).

\section{Conclusiones}

- La metodología propuesta permite integrar la normatividad establecida dentro del plan de ordenamiento territorial, en lo referente a la reglamentación de usos del suelo y los tratamientos urbanísticos, con los procesos de formación y actualización catastral, en los cuales se utilizan los estudios de zonas homogéneas físicas urbanas.

- La incorporación de estas dos nuevas variables (Áreas de actividad y tratamientos urbanísticos), garantizan la determinación de zonas homogéneas geoeconómicas, más técnica y justas para el cálculo del avalúo catastral.

- Un aporte significativo lo hace la descripción más detallada en la tipificación de todos los destinos económicos, en la metodología vigente este aspecto solo se utiliza para el destino económico residencial.

- La metodología planteada obliga a los ejecutores de los diseños de zonas homogéneas físicas y geoeconómicas a permanecer actualizados con respecto a las reglamentaciones urbanísticas, aspecto clave para el inicio de la investigación económica.

- La aplicación de esta metodología en sus dos primeras variables (Áreas de actividad y tratamientos urbanísticos) puede variar de un municipio a otro, por lo tanto se recomienda leer y analizar correctamente cada uno de los planes de ordenamiento territorial vigentes de los municipios que se van a trabajar catastralmente.

- Con esta nueva información incorporada de los POT municipales a los procesos catastrales, se gana información que puede servir para la planeación y desarrollo municipal del municipio en cuestión.

- Se recomienda a la Subdirección de Catastro del Instituto Geográfico Agustín Codazzi, adoptar esta metodología cuanto antes en sus procesos de formación $y / 0$ actualización catastral. 


\section{Bibliografía}

Buitrago Bermúdez, Oscar. Análisis de factores formadores del precio de la tierra rural en los municipios contiguos a Bogotá. En: Revista Perspectiva Geográfica No. 6 de 2001. Universidad Pedagógica y Tecnológica de Colombia. pp. 67-103

Cadena Cepeda, Hugo, Jiménez David y Suárez Pinilla Elías. Actualización de la Metodología en la determinación de zonas homogéneas físicas, mediante la utilización de un SIG caso estudio: municipio de Zipaquirá zona urbana. Tesis de grado para optar al título de Especialista en Sistemas de Información Geográfica. Convenio Universidad Distrital - IGAC, Bogotá, 2001.

Concejo Municipal de Zipaquirá. Acuerdo 12 de 2000.

Rodríguez C., María Luisa. El valor del suelo y Competencia por la ocupación espacial. Área de influencia de la autopista Norte (Calle 200-Briceño) en Revista Perspectiva Geográfica No. 6 de 2001. Universidad Pedagógica y Tecnológica de Colombia. pp. 105-116. 
